\title{
Democratic Process in Online Crowds and Communities
}

\author{
Caroline Haythornthwaite
}

School of Library, Archival \& Information Studies, The iSchool at UBC, University of British Columbia, 470-1961 East Mall, Vancouver, BC, Canada V6T 1Z1, c.haythorn@ubc.ca

\begin{abstract}
This paper explores the underlying structures that support participation and reputation in online crowd and community-based peer productions. Building on writings on open source, peer production, participatory culture, and social networks, the paper describes crowd and community structures as two ends of a continuum of collective action - from lightweight to heavyweight - differentiated by the extent of connectivity and engagement between contributions and among contributors. This is followed by an examination of the recognition, reputation and reward systems that support these collectives, and how these affect who controls and who contributes information. The aim of this exploration is to gain insight for understanding motivations and structures for e-participation in these different, potentially democratic, forums.
\end{abstract}

Keywords: Crowds, communities, peer production, social networks, participation, collective action, online communities, crowdsourcing

Acknowledgement: Papers on lightweight/crowdsourced and heavyweight/community-sourced peer production have been presented at the Hawaii International Conference on System Sciences (2009), at the Knowledge Communities conference held in Reno, Nevada at the Center for Basque Studies (2009), as part of the Leverhulme Trust Public Lecture series on 'Learning Networks' in 2009-10, and at the e-Democracy conference (CeDEM) at Krems, Austria in May 2011. Thanks go to participants at these events for their comments and support in pursuing these ideas.

n 2005, Bill Gates wrote that we now live in an "information democracy" made possible by the information freely available on the Internet, but adds that "while we've gone a long way toward optimizing how we use information, we haven't yet done the same for knowledge" (p.84). While there is still a long way to go in devising tools to enhance ways of extracting knowledge from the vastness of the Internet information space, the collective action of individuals worldwide is already at work on the problem. We are seeing now the emergence of many ways in which knowledge collectives are forming on the Internet, providing structure, focus and communities that add meaning to information. While online communities were the surprise of the first wave of online organizing, crowds are the more recent manifestation of collective action. Meanwhile, individual presence online has evolved from having a web page, as marked by the first Web 1.0 wave of online participation, to the kind of relational visibility and interactivity that marks Web 2.0 contribution and participation. Of great interest in terms of information democracy are the patterns and configurations within that sphere that affect equity in that democracy. Why, for example, do some contributions gain greater visibility than others? What reputation and recognition systems are being enacted online that shape the information landscape? And, what are the implications of these configurations for a voice in such a democracy?

Among the many emerging forms of organizing online are the two extremes of crowds and communities. Elsewhere I have articulated a set of organizing principles that distinguish crowds and communities, yet place them at two ends of a continuum (Haythornthwaite, 2009, 2011). The work builds on research about structures and motivations associated with open source (Raymond, 1999), peer production (Benkler, 2006), and participatory culture (Jenkins, 2006), as well as drawing on my own work and that of others on structural aspects of social networks both offline and online and motivation for contribution in crowds and communities (Wasserman \& Faust, 1996; 
Scott \& Carrington, 2011; Wellman et al, 1996; Haythornthwaite, 2005, 2008; Budhathoki, 2010). This examination of the structures that support online crowds and communities suggested a continuity in knowledge organizing, with crowdsourced examples falling at what I have referred to as a 'lightweight' end of contributory behavior and community-based examples at a 'heavyweight' end (where weight refers to the commitment to the collective rather than to the importance of the work). I chose the terms lightweight and heavyweight to avoid connotations associated with other terms for such collectives, such as crowds, cooperatives, collaborations, communities, and peer productions, as well as to avoid associations with common examples such as Wikipedia which is crowdsourced yet contains in its Talk pages structural aspects of communities. My interest in these forms is in teasing out the organizational and motivational structures that underpin collective forms of organizing, leaving the judgment of collaboration, community and democracy to be addressed through evaluation of what a particular collective has actually enacted.

This paper uses this attention to organizational forms and motivations to suggest what is affecting the implementation of an information democracy. The paper begins with a recap of the ideas of lightweight and heavyweight peer production as outlined in earlier papers. This is followed by an examination of the recognition, reputation and reward systems that support these collectives, and how these affect who controls and who contributes information. The aim is to gain insight for understanding e-participation in these different, potentially democratic, forums.

\section{Lightweight and Heavyweight Forms of Organizing}

Online crowds and communities describe two very different forms of organizing. The former harnesses the reach of the Internet and the ubiquity of access to draw in contributions from many different individuals. In keeping with the idea of crowds, we expect a lot of contributors, all moving toward the same goal, maintaining attention to an event external to themselves - the crowd at a football game, demonstrators at a political rally. We also expect the crowd to disperse as the event ends, moving on individually to other pursuits. In keeping with the idea of the Internet, we expect common access, and easy submission toward an overall goal. We can also add a longer timeframe to online crowds as collective action is not chained to a real-time event or to a co-located critical mass of participants. And, in keeping with organizational production, we expect similar contributions, anonymous and independent, that together support a larger enterprise, as in the piece work that sustains a manufacturing operation.

By contrast, in keeping with the idea of communities, we expect a more limited set of contributors, committed to each other, the community, and the goals of that community. In keeping with group behavior, contributors belong because they possess some credential, whether that is as simple as living in the same geographical neighborhood or common membership in an organization, or a more complicated set of acquired credentials, such as that necessary to claim membership in the medical or academic community. By contrast with crowds, communities demonstrate diversity in roles and niches; tasks and inputs differ, reflecting expertise and differentiation among contributors; outcomes depend on interconnection of tasks and people that serve and create the community as a whole. The timeframe for a community extends beyond a single event, and beyond the participants themselves; communities maintain a structure where individuals may enter and exit, yet the whole retains its shape and purpose.

There are many more attributes to describe crowds and communities. But since, as noted above, the terms carry such established connotations, to articulate the attributes without labeling the resulting form, I switch now to using the lightweight to heavyweight continuum as a way to address structures rather than extant examples of online collectives.

Figure 1 provides a visual depiction of the light- to heavyweight forms of collectives. In brief, the lightweight end of the figure describes the most instrumental of crowdsourced enterprises. Examples at this extreme lightweight end include participation in distributed computing efforts that allow access to a participant's idle computer cycles, such as applications that use the Berkeley Open Infrastructure for Network Computing (BOINC), e.g., SETI@home. Also at the lightweight 
end are sites that ask for human action that requires little learning or apprenticeship. Examples include GalaxyZoo (http://galaxyzoo.org) which asks contributors to answer a series of questions about galaxy shapes based on what they see in an image of a galaxy; and Distributed Proofreaders (http://www.pgdp.net/c/; also discussed in Benkler, 2006) which asks individuals to proof read and correct texts that have been created using optical character recognition (OCR). A similar venture in the commercial realm is Mechanical Turk which asks contributors to complete small tasks for small amounts of money (e.g., 2 cents a response). Moving a little further along the lightweight end, we can add in sites like 23andWe where participants who have already submitted a sample for genomic testing (23andMe) are invited to participate in surveys that are aggregated for research studies of genetics. And still at the relatively lightweight end, we find the contributory portions of online wikis, such as Wikipedia for general knowledge, and OpenStreetMap for geographical data.

Crowd-sourced, Lightweight

- Contributions

- Many, simple, discrete, unconnected

- Anonymous, impersonal

- Contributors

- Many, lightly-tied non-networked individuals

- Control

- External to contributors

- Motivation

- Coorientation to common purpose

\section{Community-sourced, Heavyweight}

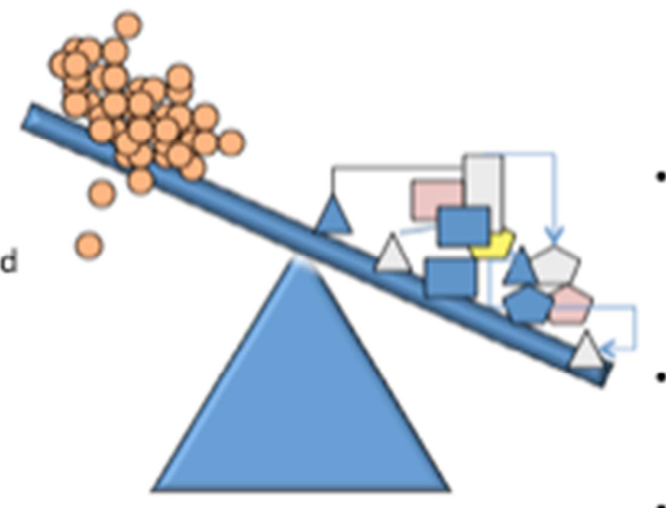

- Contributions

- Fewer, diverse, connected

- Named, visible attribution

- Contributors

- Fewer, heavily. tied, networked individuals

- Control

- Internal to community

- Motivation

- Overall purpose and to group interaction

Figure 1: Lightweight to Heavyweight Collectives

What is common across these and similar lightweight peer productions is that it takes little to be allowed to contribute: little time to learn how to contribute and little time to make the contributions, with minimal rewards for participation. As depicted in Figure 1, contributions are basically the same, and they are separate and distinct. No coordination is required with other contributors, nor with previous contributions; each task is independent and does not depend of completion of earlier tasks. This is piece work, completed by independent contractors, unknown to each other, but tied by common connection to the site or activity on the site.

By contrast, the heavyweight end describes collectives where participants are tied both by the connection to the site or its purpose and by the connection to each other. These collectives distinguish involvement by meeting strong requirements for credentials either developed within the community, as novices become experts, or by invitations to join extended to those considered already qualified. Becoming a recognized member of the collective requires learning and adopting 
community practices. Tasks are completed in concert with others, and with awareness and attention to the work, opinions and practices of others. These collectives are exemplified by virtual communities, online discussion groups, distributed teams, and collaborative research groups. Through processes of group awareness, combined with attention to the motivation for the group's existence, norms and practices are developed and followed that support group processes. Among the processes that members of heavyweight peer productions learn and practice are proper forms of discourse, collaboration, and communication (Miller, 1994; DeSanctis \& Poole, 1994; Orlikowski, 2002; Haythornthwaite, Lunsford, Bowker \& Bruce, 2006; Haythornthwaite, 2006).

What is common across heavyweight collectives it that it takes time to become and be known as a member; and it takes adherence to norms to remain a member. Through observation of other members' actions, individuals gain and act in the group based on their understanding of others place in the collective, their own expertise (and thus, their place in the collective), and the overall group structure (i.e., the places of others in the collective). Individuals gain transactive memory, such as knowing who is the expert on what, who knows what, or who is friends with whom (Wegner, 1987; Krackhardt, 1987; Moreland, 1999; Hollingshead, Fulk and Monge, 2002). Such knowledge helps individuals locate as well as allocate information in the network, supporting the differentiation of individuals and their roles in the group. These actions build the social capital of the group, which includes group practices in support of information and knowledge retention and mobilization (Cohen \& Levinthal, 1990; Lin, 1999; Burt, 2000; Haythornthwaite, 2010). Social capital can also include the process through which community norms are policed and controlled, from group policing that shuns trolls and off-topic discussion (McLaughlin et al, 1995), to contracts that provide trust in community practices through the support of legal structures (Burt, 2000).

After many years of research on virtual communities, it is generally accepted that these kinds of heavyweight communities can and do exist online, and can be found in e-learning groups, academic discussion groups, online multi-player games, social support groups, and more. Over the years, many authors have articulated the differences between offline and online groups, notably in asynchronous participation, lack of visual presence of members, etc.; yet, community norms have emerged over and over again, adapted and transformed to the venue of computer-mediated communication (e.g., Baym, 1995; Jones, 1995; McLaughlin, Osborne, \& Smith, 1995; Haythornthwaite, Kazmer, Robins \& Shoemaker, 2000; Kendall, 2002; Preece \& MaloneyKrichmar, 2005; Haythornthwaite, 2005). We are at the point now where debating whether community can exist online is not as important as articulating what makes a better or worse community online, and how to keep up with its evolving forms (Haythornthwaite, 2007).

While virtual communities demonstrate the heaviest end of the continuum, wikis provide structures for community discussion that support a middle ground on communities. Their combination of front-stage information input and revision can be balanced by a back-stage discussion and debate on proper posting as well as interpersonal connection. They remain a middle ground because of the limited scope of attention, e.g., with attention to the specific application (encyclopedia or mapping system), or information point(s) for participation (e.g., to the specific entry or range of entries, or to specific regions for mapping). Similarly in online support groups, lists and discussions, when the orientation to a condition, profession, region or purpose is narrow and remains narrowly observed, the collective demonstrates a middle ground between light and heavyweight organizing. Discussion and internal recognition of actors exists, but remains concentrated somewhat instrumentally on the topic at hand.

\section{Social Network Connectivity}

Underpinning these ideas of light and heavyweight peer productions are the principles of social networks and social network ties, and the discussions that follow build on these principles to explain lightweight (crowd) and heavyweight (community) behaviors. Such peer productions rest on contributions by individuals to a collective whole, with interpersonal interactions ranging from minimal, highly instrumental connections to frequent, multi-topic, multi-purpose, and emotional 
connectivity. Studies of social networks have given us a vocabulary and set of techniques for addressing collectives through the social connections between actors rather than through aggregate behavior (e.g., Scott \& Carrington, 2011). In social network terms, actors maintain relations (e.g., working together, socializing) that form ties (work ties, friendship ties), across a population of interest. These ties reveal the configurations of interaction that define the social network (e.g., work relationships among members of an organization). The nature of ties is important for the discussion here. Ties vary in strength from weak to strong, with strength discussed instrumentally in terms of number and types of relations maintained, frequency of interaction and reciprocity in exchanges, and, less instrumentally, in terms of the importance (to the actors) of the relations maintained, and the intimacy of the relationship.

The first observation in relation to the light- and heavyweight models is that a direct connection between contributors in the lightweight model is not required. Individuals add to a peer production without the need to interact with other contributors or with others' contributions. However, at this very light end, we can observe a connection through use of the site. Thus, two actors may be considered to be tied (very weakly) because they make contributions to the same site. This type of connection is derived from the idea of common exposure to information, and hence the development or pre-existence of a common orientation to a subject. Thus, if we read the same book (as in many city reading programs, e.g., Chicago's 'One Book, One Chicago'), we are exposed to the same ideas and thus have some common ground on a subject. It adds to the homophily between actors. Taking the same idea to crowdsourcing, we can make the assumption that individuals who contribute to a common project or site have some commonality in their attitudes or interests. Such is the basis for saying here that participants in lightweight collectives are tied by their common connection to the peer production, and by their coorientation to its purpose (Chaffee \& McLeod, 1973).

Heavyweight peer productions demonstrate strong tie structures between actors in the network. Strong ties are multiplex, i.e., built on multiple points of commonality and interaction, supported through engagement in multiple relations, use of multiple forums for discussion, and reciprocity in exchanges. Notable for heavyweight collectives is that the ties are interpersonal, maintained between actors in the network. Of particular salience for distinguishing between lightweight and heavyweight peer productions is that visibility of actors and their individual and network interactions is necessary to build and sustain strong tie networks. Visibility builds the community as a recognizable structure to others, and also builds internal structures that define the collective, and, in the case of self-perpetuating networks, supports the collective independently of the individuals involved (i.e., creating persistent social structures that transcend individual membership). Visibility allows newcomers to a community to see and follow norms. Note that not all members of a heavyweight peer production or a community need to be strongly tied to every other member: novices, lurkers, and occasional contributors are all important for maintaining a community longterm. The point is that a core heavyweight structure must be present for the whole network to exist and to continue to exist, and that the structure needs to be continuously reinforced through adherence to and enactment of social norms (Bruckman \& Jensen, 2002; Contractor \& Eisenberg, 1990; DeSanctis \& Poole, 1994). Such a structure allows for visibility, contribution diversity, role differentiation, community monitoring and regulation, and reputation, recognition and reward in accordance with community norms.

\section{Participation in Crowds to Communities}

New forms of organizing that bring in crowds of strangers or support distributed communities of interest raise questions about individual motivations and perspectives on participation and engagement in these emerging democratic forms. As contributing online becomes technologically easier - whether clicking to give the 'thumbs-up' Like to a favorite posting, blogging on a topic of interest, or contributing to wikis - the question remains of why individuals take the time and effort to make such a contribution. Theories and research on individual motivations about such behaviors are beginning to emerge, often with the aim of supporting frameworks for the design of social or 
commercial online collective efforts. Diversity in these enterprises is such that terms such as 'community', with its connotations of common ground and common goals, and 'democracy', with connotations of equal and open participation, are judgments that should only be applied post hoc, i.e., after evaluation of what the collective activity has been enacted. We also need to consider the tipping points of where collective action, e.g., in crowds, becomes a collective movement, e.g., in communities.

As above, to separate judgment of outcomes from structures, I continue to use the terms lightweight and heavyweight to help distinguish forms of organizing from outcomes. Building on the position outlined above, the remainder of this paper turns to consideration of the motivators and recognitions that provide the momentum for participation at the two ends of this continuum. A wide interpretation of participation is taken in keeping with ideas of an information democracy, ubiquitous learning, and participatory culture (Gates, 2005; Jenkins, 2006; Peters, 2007; Cope \& Kalanzis, 2009). The discussion aims to articulate the array of participatory behaviors that support democratic activity - from a single-click vote in a lightweight structure to long-term engagement and debate in a heavyweight peer production.

\subsection{Motivation}

The Internet beckons as a forum for the open exchange of information and ideas. Yet, as initiatives for collective action appear, the question remains of why individuals will spend their time contributing to these efforts and/or how to motivate individuals to contribute. Mechanical Turk uses minor monetary rewards to gain attention, but what do other sites provide? In discussion of open source and open access contribution, writers such as Raymond (1998, 1999), Benkler (2005, 2006), and Willinsky (2005) all point to the dual motivation of a personal-but-shared everyday need as motivation for contribution to peer productions. Adapting this idea to the continuum of light- to heavyweight forms of organizing, we can map onto this a range of commitment to sharing related to the need, the commitment to the collective as the forum for addressing the need, and the personal engagement in the processes that support addressing the need.

In lightweight initiatives, the individual who contributes does not interact directly with others, and their contribution to a larger goal is mediated through the structures and intent of the site organizers. They cannot modify the rules of the game except by not playing. To engage they must trust in - or not care about - the use site owners make of what they contribute. The sites themselves must appeal to something greater than the reward for local contribution. As noted above, coorientation to an purpose even beyond the goals of the site organizers can act as a major motivator. Here, for example, is a comment on contributing mapping data to Google after the earthquake in Haiti:

Yes, I generally don't want to just give my data to Google without getting anything back and so yes I am a strong supporter of a share-alike license normally. But the reason I want a sharealike license is because I don't want to work for free and want to get paid for my work. Not with money but normally with more data. However here in Haiti, my payment would be that this work might save lives or at least help make it less devestating [sic] for some. This is more than Google can ever give back! [Comment from amm at Fri, 22 Jan 2010 23:10:28 +0000; emphasis added] (http://www.openstreetmap.org/user/Harry\%20Wood/diary/9332)

Motivation can also be generated as crowds engaged in an instrumental activity create and add their own structures that support a more personal connection and strengthen the relationship with the instrumental enterprise. For example, the 'DC Vault' compiles statistics on contributions to distributed computing applications (such as SETI), inviting participants and teams to show off their scores:

The DC Vault is the place to compare your team's performance against others, the place to look out for when you plan your next taunting fest, the place you can refer others to and brag about how devilishly high ranked your team is ... or not. 
Compare your team's performance and ranking in many different categories or look at the overall picture, highlight your team of choice, compare teams at a glance, anything is possible with the links above! (Retrieved March 21, 2011 from: http://www.dcvault.com/index.php)

Motivation can start as an orientation to an activity, such as gaming, but be supplemented through internal mechanisms that support a more heavyweight form of interaction. This comment about structures in a game environment describes just such a development:

Like most games, the ones produced by Zynga and its peers appeal to people's natural competitive instincts. Leader boards and a host of other features allow players to show off their status within a game to their friends. But the games also encourage lots of co-operation among players, who build rapport by, say, sending virtual gifts to each other or handing virtual currency to new players when they join a game. "The best virtual goods have real currency," says Mark Pincus, Zynga's boss. He reckons that the games have become so popular because they combine fun with the various ways to strengthen relationships that Facebook and other networks have brought online. (Economist, January 30, 2010, p. 13-14)

Comments in the quote above shows aspects of crowd/lightweight engagement in the common orientation to competitive activity ('appeal to people's natural competitive instincts'); community/heavyweight engagement in attention to others ('show off their status', 'encourage lots of co-operation among players', and 'build rapport'); and crowd plus community aspects as attention to fun is combined with building relationships: 'the games have become so popular because they combine fun with the various ways to strengthen relationships.'

A recent study of OpenStreetMap, an open source, voluntary geographic information system, also shows motivation that differs between those engaging in a lightweight fashion with the system (a category of casual mappers) and those contributing in a more heavyweight fashion (serious mappers, defined as at least two standard deviations from the mean or higher on one or more of: number of nodes contributed (excluding mass uploads), length of contribution period, and frequency of contribution; Budhathoki \& Haythornthwaite, in press). The study found that casual mappers reported higher motivation scores relating to the open source nature of the project than did serious mappers, e.g., in Likert scale assessments of the statements: 'It is important to help others by providing digital maps that are available for free'; and 'Digital map data should be available for free only for non-commercial applications'. By contrast, serious mappers reported higher motivations for items relating to community, including 'OSM community is important to me', and 'I want to be recognized as an active OSM contributor'.

Bound into these orientations and motivations, and to what can seem like side activities (e.g., fun), are aspects of recognition, reputation and reward that underpin lasting ties to others and to the peer production as a whole. The next section explores these in more detail.

\subsection{Recognition, Reputation and Reward}

Reputation is build on visibility of contributions. Hence, it is immediately apparent that the kind of anonymous, non-differentiated contribution that defines a lightweight collective provides very lean mechanisms for building a reputation. No identification of the individual is needed for contributions to count in a crowdsourced production, e.g., it doesn't matter if you are identified for your contribution, nor if you sign in under different names each time. Indeed, avoiding a reputation may be the very motivator for contributing, e.g., as individuals can add and edit without embarrassment about potential errors or inappropriate posting. Because each contribution is essentially identical ('Like' votes, clicks, computer cycles), what reputations are created can only address contributions quantitatively, e.g., the number of items submitted, tasks completed, clicks made, votes cast, or links to a website. A contributor to a lightweight collective may also only be able to judge the worth of their contribution by a numeric count of its use: Huberman, Romero and Wu (2009) found that as the numbers of downloads of their YouTube videos fall off, contributors stop adding videos. Thus, 
overall, an essential element of lightweight recognition, reputation and reward systems is that these are able to be evaluated on a quantitative assessment, e.g., number of downloads, rather than a qualitative assessment, e.g., comments on the video.

By contrast, heavyweight collectives operate through visibility and continuity in individual persona, and reputations are built on the recognition of or by others in the network. The DC Vault example above shows how a lightweight endeavor may add reputation (or have reputation added) to its features. Yet, there is still a distinction between the basic construction of the lightweight production mechanism where individual identity does not matter, and the heavyweight mechanism where identity is vital. Reputation grows from the recognition given by community members to the contributions of individuals, and is an indicator of successful conformity to community standards and norms. But, perhaps more significantly, the value of such recognition varies and depends on the status of the individual giving the recognition. In a heavyweight collective, it matters who finds the contribution and comments on or uses it. Reputation depends not only on instrumental, countable aspects of recognition, e.g., number of links, but also on who is recognizing whom, i.e., what site links to yours. Consider for example the difference between a rave review of an academic book when given by a student versus a senior scholar. The voice of the latter carries more signficance and garners further recognition for the author than voice of the former. Recognition from a high-ranking member of a network about a contribution enhances the reputation of the contributor beyond the value of a straightforward count of citations. By contrast, a cite from a lowranking member to a high-ranking one adds very little to the latter's existing reputation; such recognition may carry no more significance than a quantitative measure of contribution. To have members sufficiently distinguished by ranks to make their attributions have this kind of differential influence requires a heavyweight structure and long-lasting acceptance and conformity to rank and reputation norms. Lightweight systems only have the option of using rank-free ties, because no basis for rank can exist in a truly lightweight production system.

Reputation is a network effect. It is a form of social capital, that emerges from the actions and attentions of members of a social network to other members of the network, dependent on the structures they build and enact. A major difference then between lightweight and heavyweight systems is that the former have no internal mechanisms for enacting reputation. If these are to exist, even at a basic quantitative level, they must be designed into the system by designers and operators of such systems. Thus, recognition and reputation are under the control of authorities beyond the individual contributor. At the heavyweight end, it is human, collegial evaluation that provides the most relevant feedback, with evaluations emerging from and reinforcing community values. While both lightweight and heavyweight production require contribution by peers, the reputation and recognition system for lightweight collective contribution operates outside the control of the peer group, while for heavyweight collective the system it operates inside the peer group. Overall, lightweight recognition systems can only address contributions, whereas heavyweight systems can address both contributions and contributors (see also Duguid, 2006), and the networks they form.

\subsection{Implications for Democratic Process}

This brief and by no means complete evaluation of structures that support and are emerging for new forms of organizing raise questions about how such organizing can or will be used in democratic processes. Some questions that arise around e-voting are: Will voters approach such online activity as a crowd or community based initiative? What differences will it make if they perceive voting and participation as a crowd activity (vote and leave) or a community activity (vote and stay to sustain the outcome)? Will this be reflected in differences in individual participation rates, sense of obligation to vote, or engagement in debate before or after voting? Will there be differences in framing debate, i.e., modeling a lightweight or heavyweight approach? What outcomes can be expected in participant demographics, knowledge of issue, or engagement with issues? 
Some further questions arise around new roles for participants in sociotechnical, participatory cultures and e-democracy. Are we in or moving to an 'information democracy', and if so, what mechanisms of reputation are affecting what information gains supremacy, trust? Already we know that offline structures affect who can and does contribute to the information cyberscape (e.g., see Crutcher \& Zook, 2009, who report how information online more greatly represented affluent areas of New Orleans after Hurricane Katrina than poorer areas). Further, what tensions exist between interests of the individual actor, and the actor in the network, and the desire and use of anonymity versus named, attributed contribution? How will this affect contribution and participation online?

This paper has outlined just a beginning to examination of structural features that distinguish commitment, trust and contributory behavior in crowd and community collective structures. As participation, both formal and informal, turns to online, internet-based collective forums, the more important it is to understand the facilities and constraints of the different organizing systems and the effects these have on e-participation, voting, information sharing and democratic process.

\section{References}

Baym, N. K. (1995). The emergence of community in computer-mediated communication. In S. Jones (Ed.), Cybersociety: Computer-mediated Community and Communication (pp. 138-163). Thousand Oaks, CA: Sage.

Benkler, Y. (2005). Common Wisdom: Peer Production of Educational Materials. COSL (Center for Open and Sustainable Learning), Utah State University Press. Retrieved March 23, 2011 from: http://www.benkler.org/Common Wisdom.pdf

Benkler, Y. (2006). The Wealth of Networks: How Social Production Transforms Markets and Freedom. New Haven, CT: Yale Univ. Press.

Bruckman, A. \& Jensen, C. (2002). The mystery of the death of Mediamoo: Seven years of evolution of an online community. In K. A. Renninger \& W. Shumar, Building Virtual Communities: Learning and Change in Cyberspace (pp. 21-33). Cambridge: Cambridge University Press.

Budhathoki, N. R. (2010). Participants' Motivations to Contribute Geographic Information in an Online Community. Unpublished doctoral dissertation, Univ. of Illinois at Urbana-Champaign, Champaign, IL. [Available online at: http://hdl.handle.net/2142/16956]

Budhathoki, N. \& Haythornthwaite, C. (in press). Motivation for open collaboration: Crowd and community models and the case of OpenStreetMap. American Behavioral Scientist.

Burt, R.S. (2000). The network structure of social capital. Research in Organizational Behavior, 22, 345-423.

Chaffee, S. \& McLeod (1973). Interpersonal perception and communication. American Behavioral Scientist, 16(4), 483-488.

Cohen, W.M. \& Levinthal, D.A. (1990). Absorptive capacity: A new perspective on learning and innovation. Administrative Science Quarterly, 35, 128-152.

Contractor, N. S. \& Eisenberg, E. M. (1990). Communication networks and new media in organizations. In J. Fulk \& C. W. Steinfield. (Eds.), Organizations and Communication Technology (pp. 143-172). Newbury Park, CA: Sage.

Crutcher, M. \& Zook, M. (2009). Placemarks and waterlines: Racialized cyberscapes in post Katrina Google Earth, GeoForum, 40(4): 523-534.

DeSanctis, G. \& Poole, M.S. (1994). Capturing the complexity in advanced technology use: Adaptive structuration theory. Organization Science, 5(2), 121-47.

Duguid, P. (2006). Limits of self-organization: Peer production and 'laws of quality'. First Monday, 11(10). Retrieved March 23, 2011 from: http://firstmonday.org/issues/issue11 10/duguid/index.html

Gates, B. (2005). The new road ahead: How 'intelligent agents' and mind-mappers are taking our information democracy to the next stage. Newsweek, p. 84.

Haythornthwaite, C. (2006). Facilitating collaboration in online learning. Journal of Asynchronous Learning Networks, 10(1). Retrieved March 23, 2011 from: http://www.sloan-c.org/publications/jaln/index.asp

Haythornthwaite, C. (2007). Social networks and online community. In A. Joinson, K. McKenna, U. Reips \& T. Postmes (Eds.), Oxford Handbook of Internet Psychology (pp. 121-136). Oxford, UK: Oxford University Press.

Haythornthwaite, C. (2010). Social networks and information transfer. In M.J. Bates \& M.N. Maack (Eds.), Encyclopedia of Library and Information Sciences, third edition, 1:1, 4837-4847. NY: Taylor \& Francis.

Haythornthwaite, C. (2011). Online knowledge crowds and communities. In Knowledge Communities. Reno, NV: Center for Basque Studies. [Available online at: http://hdl.handle.net/2142/14198] 
Haythornthwaite, C. (Ed.) (2005). Computer-Mediated Collaborative Practices and Systems. Journal of Computer-Mediated Communication, 10(4), articles 11-18. Retrieved March 23, 2011 from: http://jcmc.indiana.edu/vol10/issue4/

Haythornthwaite, C. (Jan. 2009). Crowds and communities: Light and heavyweight models of peer production. Proceedings of the 42nd Hawaii International Conference on System Sciences. Los Alamitos, CA: IEEE Computer Society. [Available online at: http//hdl.handle.net/2142/9457]

Haythornthwaite, C., Kazmer, M. M., Robins, J. \& Shoemaker, S. (2000). Community development among distance learners: Temporal and technological dimensions. Journal of Computer-Mediated Communication, 6(1). Retrieved March 23, 2011 from: http://jcmc.indiana.edu/vol6/issue1/haythornthwaite.html

Haythornthwaite, C., Lunsford, K.J., Bowker, G.C., \& Bruce, B. (2006). Challenges for research and practice in distributed, interdisciplinary, collaboration. In C. Hine (Ed.), New Infrastructures for Science Knowledge Production (pp. 143-166). Hershey, PA: Idea Group.

Hollingshead, A. B., Fulk, J., and Monge, P. (2002). Fostering intranet knowledge sharing: An integration of transactive memory and public goods approaches. In P. Hinds and S. Kiesler (Eds.), Distributed Work: New Research on Working Across Distance Using Technology. Cambridge, MA: MIT. pp. 335-355.

Huberman, B.A., Romero, D.M. \& Wu, R. (2009). Crowdsourcing, attention and productivity. Journal of Information Science, 35(9), 758-765.

Jones, S. G. (1995). Understanding community in the information age. In S.G. Jones (Ed.), CyberSociety: ComputerMediated Communication and Community (pp. 10-35). Thousand Oaks, CA: Sage.

Kendall, L. (2002). Hanging out in the Virtual Pub: Masculinities and Relationships Online. Berkeley, CA: Univ. of California Press.

Krackhardt, D. (1987). Cognitive social structures. Social Networks, 9, 109-134.

Lin, N. (1999). Building a network theory of social capital. Connections, 22(1), 28-51.

McLaughlin, M. L., Osborne, K. K. \& Smith, C. B. (1995). Standards of conduct on usenet. In S.G. Jones (Ed.), CyberSociety: Computer-Mediated Communication and Community (pp. 90-111). Thousand Oaks, CA: Sage.

Miller, C. (1994). Rhetorical community: The cultural basis of genre. In A. Freedman \& P. Medway (Eds.), Genre and the New Rhetoric (pp. 67-78). Basingstoke, UK: Taylor \& Francis.

Moreland, R. (1999). Transactive memory: Learning who knows what in work groups and organizations. In L. Thompson, J. Levine, and D. Messick (Eds.), Shared Cognition in Organizations. Mahwah, NJ: Lawrence Erlbaum. pp. 3-31.

Orlikowski, W. J. (2002). Knowing in practice: Enacting a collective capability in distributed organizing. Organization Science, 13(3), 249-273.

Preece, J., and Maloney-Krichmar, D. (Eds.)(2005). Online communities. Journal of Computer-Mediated Communication, 10(4), articles 1-10. Retrieved March 23, 2011 from: http://jcmc.indiana.edu/vol10/issue4/

Raymond, E. (1998). The cathedral and the bazaar. First Monday, 3(3). http://www.firstmonday.dk/issues/issue3 3/raymond/

Raymond, E. S. (1999) The Cathedral \& the Bazaar: Musings on Linux and Open Source by an Accidental Revolutionary. Cambridge, MA: O’Reilly.

Scott, J. \& Carrington, P. (Eds.)(2011). Handbook of Social Network Analysis. London: Sage.

Wegner, D. (1987). Transactive memory: a contemporary analysis of the group mind. In B. Mullen \& G. Goethals (Eds.), Theories of Group Behavior (pp. 185-208). New York: Springer-Verlag.

Willinsky, J. (2005). The unacknowledged convergence of open source, open access, and open science. First Monday, 10(8). Retrieved March 23, 2011 from: http://firstmonday.org/issues/issue10 8/willinsky/index.html.

\begin{abstract}
About the Author
Caroline Haythornthwaite

Caroline Haythornthwaite is Director and Professor, School of Library, Archival and Information Studies, The iSchool at the University of British Columbia. She joined UBC in 2010 after 14 years at the University of Illinois at Urbana-Champaign, where she was Professor in the Graduate School of Library and Information Science. In 2009-10 she was Leverhulme Trust Visiting Professor at the Institute of Education, University of London presenting and writing on 'Learning Networks', and in summer 2009 she was a visiting researcher at the Brazilian Institute for Information in Science and Technology (IBICT), Rio de Janeiro, Brazil. She has an international reputation in research on information and knowledge sharing through social networks, the development and nature of community online, the nature and constraints of interdisciplinary collaboration, and the transformative effects of the Internet and web 2.0 technologies on learning and collaborative practices. Major publications include The Internet in Everyday Life (2002, with Barry Wellman); Learning, Culture and Community in Online
\end{abstract}


Education (2004, with Michelle M. Kazmer), the Handbook of E-learning Research (2007, with Richard Andrews), and Elearning Theory and Practice (2011, with Richard Andrews). 